TITLE:

\title{
Pair-correlation entropy of hydrophobic hydration: Decomposition into translational and orientational contributions and analysis of solute-size effects
}

\section{$\operatorname{AUTHOR}(\mathrm{S}):$}

Kinoshita, M; Matubayasi, N; Harano, Y; Nakahara, $\mathrm{M}$

\section{CITATION:}

Kinoshita, M ...[et al]. Pair-correlation entropy of hydrophobic hydration: Decomposition into translational and orientational contributions and analysis of solute-size effects. The Journal of Chemical Physics 2006, 124(2): 024512.

ISSUE DATE:

2006-01-14

URL:

http://hdl.handle.net/2433/50434

\section{RIGHT:}

Copyright 2006 American Institute of Physics. This article may be downloaded for personal use only. Any other use requires prior permission of the author and the American Institute of Physics. 


\title{
Pair-correlation entropy of hydrophobic hydration: Decomposition into translational and orientational contributions and analysis of solute-size effects
}

\author{
Masahiro Kinoshita ${ }^{\text {a) }}$ \\ Institute of Advanced Energy, Kyoto University, Uji, Kyoto 611-0011, Japan \\ Nobuyuki Matubayasi \\ Institute for Chemical Research, Kyoto University, Uji, Kyoto 611-0011, Japan \\ Yuichi Harano \\ Institute of Advanced Energy, Kyoto University, Uji, Kyoto 611-0011, Japan \\ Masaru Nakahara \\ Institute for Chemical Research, Kyoto University, Uji, Kyoto 611-0011, Japan
}

(Received 6 September 2005; accepted 18 October 2005; published online 12 January 2006)

\begin{abstract}
We develop an efficient method to evaluate the translational and orientational contributions to the solute-water pair-correlation entropy that is a major component of the hydration entropy. A water molecule is modeled as a hard sphere of diameter $d_{S}=0.28 \mathrm{~nm}$ in which a point dipole and a point quadrupole of tetrahedral symmetry are embedded. A hard sphere of diameter $d_{M}$, a hydrophobic solute, is immersed at infinite dilution in the model water. The pair-correlation entropy is decomposed into the translational and orientational contributions in an analytical manner using the angle-dependent Ornstein-Zernike integral equation theory. The two contributions are calculated for solutes with a variety of sizes $\left(0.6 \leqslant d_{M} / d_{S} \leqslant 30\right)$. The effects of the solute-water attractive interaction are also studied. As $d_{M}$ becomes larger, the percentage of the orientational contribution first increases, takes a maximum value at $d_{M}=D_{M}\left(D_{M} / d_{S}\right.$ depends on the strength of the solute-water attractive interaction and is in the range of 1.4-2), and then decreases toward a limiting value. The percentage of the orientational contribution reduces progressively as the solute-water attractive interaction becomes stronger. The physical origin of the maximum orientational restriction at $d_{M}=D_{M}$ is discussed in detail. (C) 2006 American Institute of Physics. [DOI: 10.1063/1.2137708]
\end{abstract}

\section{INTRODUCTION}

The hydrophobic effects ${ }^{1-15}$ play crucially important roles in a variety of physicochemical processes such as the structural stabilization of proteins, molecular self-assembly, and micelle formation. The hydrophobic hydration is one of the most fundamental subjects to be investigated. It is generally recognized that the positive hydration free energy of a nonpolar solute originates from a large, negative change in entropy upon solute insertion, especially at low temperatures. The hydration entropy, which is usually considered under the constant-pressure condition, consists of a solute-water correlation term and a water reorganization term representing the contributions of changes in water structure upon solute insertion. The solute-water correlation term represents the restrictions of the translational and orientational movements of water molecules caused by the presence of the solute. In this article, we develop an efficient theoretical method which enables us to evaluate the translational and orientational contributions to the solute-water pair-correlation entropy. In our method, the pair-correlation entropy is analytically decomposed into the two contributions. The decomposition provides physical insights into the solute-size and temperature

\footnotetext{
${ }^{a)}$ Author to whom correspondence should be addressed. Electronic mail: kinoshit@iae.kyoto-u.ac.jp
}

dependence of the hydration free energy in terms of the molecular distribution functions. We elucidate the relative importance of the translational and orientational contributions for solutes with a variety of sizes ranging from a noble-gas size to a protein-molecule size.

The first widely accepted explanation for the hydrophobicity was the "iceberg" hypothesis of Frank and Evans ${ }^{16}$ which stated that the structure of water around a nonpolar solute was highly ordered with an increase in the number of hydrogen bonds. Although they thus emphasized the importance of the water reorganization, a quantitative assessment is needed for the contributions from the water-water and solute-water correlations. ${ }^{17-22}$ Lazaridis and Paulaitis ${ }^{23}$ reported that the solute-water correlations were strong enough to account for the large, negative hydration entropy. The solvent reorganization entropy was estimated and its significance was discussed in terms of the molecular distribution functions at the initial and final states of the solute insertion. ${ }^{24-29}$ There are still uncertain and controversial aspects, but it is definite that the solute-water correlation entropy is essential in understanding the hydration entropy.

Lazaridis and Paulaitis ${ }^{23}$ reported the first attempt to evaluate relative magnitudes of the contributions from the two factors, the restrictions of orientational and translational movements of water molecules, to the correlation entropy of 
a nonpolar solute. They developed a statistical-mechanical formulation that was based on the expansion of the hydration entropy in terms of multiparticle correlation functions. The solute-water pair correlation, the leading term in the solutewater correlations, was evaluated by Monte Carlo (MC) simulations. With a factorization assumption for the paircorrelation function, they decomposed the solute-water paircorrelation entropy into the translational and orientational contributions. Their major conclusion drawn for methane is that both of the two contributions are important and the percentage of the orientational contribution is in the range of $35 \%-42 \%$ (the percentage decreases as the temperature becomes higher). The study was extended to noble gases by Lazaridis and Paulaitis ${ }^{30}$ and to small normal alkanes by Ashbaugh and Paulaitis. ${ }^{25}$ The conclusion reached in these extensions is that the percentage of the orientational contribution is dependent on the solute species and in the range of $31 \%-40 \%$ at $298 \mathrm{~K}$.

Here we apply the angle-dependent Ornstein-Zernike (OZ) relation ${ }^{31-38}$ coupled with the closure equation of the hypernetted-chain (HNC) type to the statistical-mechanical formulation derived by Lazaridis, Ashbaugh, and Paulaitis, ${ }^{23,25,30}$ and by Matubayasi et al. ${ }^{26}$ The basic equations are solved using our efficient algorithm. ${ }^{39-41} \mathrm{~A}$ water molecule is modeled as a hard sphere of diameter $d_{S}$ $=0.28 \mathrm{~nm}$ in which a point dipole and a point quadrupole of tetrahedral symmetry are embedded. A hard sphere of diameter $d_{M}$ is immersed at infinite dilution in the model water. The solute insertion is considered at $298 \mathrm{~K}$ and under the constant-pressure condition. The pair-correlation entropy is decomposed into the translational and orientational contributions in an analytical manner without using the factorization assumption. The two contributions are calculated for solutes with a variety of sizes $\left(0.6 \leqslant d_{M} / d_{S} \leqslant 30\right)$. We remark that the large solutes can be treated only by the integral equation theory. The effects of the solute-water attractive interaction are also studied. It is found that the percentage of the orientational contribution reduces progressively as the solutewater attraction becomes stronger. For the values of the parameters tested, the percentage of the orientational contribution is in the range of $10 \%-60 \%$. The orientational restriction becomes the largest at $d_{M}=D_{M}: D_{M} / d_{S} \sim 2$ in the case of no solute-water attractive interaction, and $D_{M} / d_{S}$ slightly decreases as the attraction becomes stronger. The physical origin of the maximum orientational restriction at $d_{M}=D_{M}$ is clarified by analyzing the orientational structure of water near the solute in detail.

\section{METHOD}

\section{A. Solute-water pair-correlation entropy}

The solute insertion is considered under the constantpressure condition. The solute is present in water at infinite dilution. We limit our discussion to the hydration of a spherical solute. The solute-water pair-correlation function is denoted by $g(r, \theta, \phi, \chi)$ where $r$ is the distance from the center of the solute, $(\theta, \phi)$ represents the orientation of the dipolemoment vector of water $(\theta$ is the angle between the vector and the solute-water axis), and $\chi$ describes the rotation around the dipole-moment vector. The number of water molecules whose centers are in the range $[r, r+d r]$ and the three angles are in the ranges $[\theta, \theta+d \theta],[\phi, \phi+d \phi]$, and $[\chi, \chi$ $+d \chi]$ is given by $4 \pi \rho g(r, \theta, \phi, \chi) r^{2} \sin \theta d r d \theta d \phi d \chi /\left(8 \pi^{2}\right)$ where $\rho$ is the number density of bulk water. The solutewater pair-correlation entropy ${ }^{23,25,26,30}$ defined under the constant-pressure condition $\Delta \mathbf{S}^{(2)}$ is expressed as

$$
\begin{aligned}
\Delta S^{(2)} / k_{B}= & -\left\{\rho /\left(8 \pi^{2}\right)\right\}\left[4 \pi \iiint \int g(r, \theta, \phi, \chi)\right. \\
& \left.\times \ln \{g(r, \theta, \phi, \chi)\} r^{2} \sin \theta d r d \theta d \phi d \chi\right] .
\end{aligned}
$$

In Eq. (1), $k_{\mathrm{B}}$ is the Boltzmann constant, and the integration range is $[0, \infty]$ for $r,[0, \pi]$ for $\theta$, and $[0,2 \pi]$ for $\phi$ and $\chi$. The hydration entropy depends on the triplet and higherorder solute-water correlations as well as on the pair correlation. Nevertheless, the solute-water pair-correlation entropy is certainly a major component of the hydration entropy. ${ }^{23,27-30}$

\section{B. Model}

A water molecule is modeled as a hard sphere of diameter $d_{S}=0.28 \mathrm{~nm}$ in which a point dipole and a point quadrupole of tetrahedral symmetry are embedded. ${ }^{32-34,37,38}$ The temperature is fixed at $298 \mathrm{~K}$. The effects of the molecular polarizability are taken into account using a mean-field theory. ${ }^{33}$ The effective dipole moment thus determined is approximately 1.4 times larger than the bare gas-phase dipole moment. The dimensionless dipole and quadrupole moments, $\mu_{S}^{*}=\mu_{S} /\left(d_{S}^{3} k_{\mathrm{B}} T\right)^{1 / 2}$ and $\Theta_{S}^{*}=\Theta_{S} /\left(d_{S}^{5} k_{\mathrm{B}} T\right)^{1 / 2}$, are 2.768 and 0.968 , respectively. The number density $\rho d_{S}^{3}$ of bulk water at $298 \mathrm{~K}$ is 0.7317 . The dielectric constant of this model water calculated by the reference HNC theory ${ }^{37,38}$ is 92.0. In a more rigorous model of the water molecule, a nontetrahedral quadrupole tensor and a nonzero octupole moment are introduced. ${ }^{32,33}$ Even with the present model, however, most of the important features of water can be captured. ${ }^{33}$

A hard sphere of diameter $d_{M}$, a hydrophobic solute, is immersed at infinite dilution in the model water. Ten values, $0.6 d_{S}, d_{S}, 1.4 d_{S}, 2 d_{S}, 3 d_{S}, 5 d_{S}, 10 d_{S}, 15 d_{S}, 20 d_{S}$, and $30 d_{S}$, are considered for $d_{M}$. For the solutes with $d_{M} \leqslant 3 d_{S}$ which mimic monoatomic solutes such as inert gases, the following van der Waals attractive tail is added to the hard-sphere potential

$$
u(r)=-4 \varepsilon\left(d_{M S} / r\right)^{6} \quad \text { for } r \geqslant d_{M S}=\left(d_{M}+d_{S}\right) / 2 .
$$

The values of $\varepsilon$ tested are in the range of $0-0.1 \mathrm{kcal} / \mathrm{mol}$. We note that the potential minimum in Eq. (2) is four times larger than that in the Lennard-Jones potential. The solutes with larger sizes can be regarded as simplified models of polyatomic solutes such as peptides and proteins in compact (folded) conformations, and the employment of Eq. (2) is not realistic. Therefore, for $d_{M} \geqslant 3 d_{S}$, we add the following solute-water attractive tail of the Yukawa form to the hard-sphere potential 


$$
u(r)=-4 \lambda\left(d_{M S} / r\right) \exp \left[-\kappa\left\{\left(r / d_{M S}\right)-1\right\}\right] \text { for } r \geqslant d_{M S} .
$$

That is, for $d_{M}=3 d_{S}$, both Eqs. (2) and (3) are considered. In Eq. (3), $\lambda$ controls the potential minimum and $\kappa$ the range. The values of $\lambda$ tested are in the range of $0-0.1 \mathrm{kcal} / \mathrm{mol}$, and $\kappa$ is set at 5 . We have verified that the qualitative aspects of our conclusions are not altered by the changes in these potential parameters.

\section{Theory}

We employ the angle-dependent $\mathrm{OZ}$ relation ${ }^{31-38}$ coupled with the closure equation of the HNC type for molecular fluids. A great advantage of using the integral equation theory is that the decomposition of the solute-water paircorrelation entropy into the translational and orientational contributions can be accomplished analytically without using the factorization assumption. Further, the pair-correlation function is calculated until $r \rightarrow \infty$, which is in contrast with the MC simulations.

The closure equation is expressed as

$$
\begin{aligned}
g(r, \theta, \phi, \chi)= & \exp \left\{-u(r, \theta, \phi, \chi) /\left(k_{B} T\right)+h(r, \theta, \phi, \chi)\right. \\
& -c(r, \theta, \phi, \chi)+b(r, \theta, \phi, \chi)\},
\end{aligned}
$$

where $u, h, c$, and $b$ denote the solute-water interaction potential, total correlation function, direct correlation function, and bridge function, respectively. The pair-correlation function $g(r, \theta, \phi, \chi)$ is expanded in the following manner:

$$
\begin{gathered}
g(r, \theta, \phi, \chi)=\sum_{n, \nu} g_{0 \nu}^{0 n n}(r)\left\{(-1)^{n} f^{0 n n} /(2 n+1)^{1 / 2}\right\} R_{0, \nu}^{n}(\theta, \chi) ; \\
n=0,1,2, \ldots ; \nu=-n, \ldots, n, \\
f^{0 n n}=(2 n+1)^{1 / 2},
\end{gathered}
$$

where $R_{0, \nu}^{n}(\theta, \chi)$ denotes Wigner's generalized spherical harmonics and is expressed as

$$
\begin{aligned}
R_{0, \nu}^{n}(\theta, \chi)= & (-1)^{\nu}\{4 \pi /(2 n+1)\}^{1 / 2} Y_{n}^{\nu}(\theta, \chi), \\
Y_{n}^{\nu}(\theta, \chi)= & (-1)^{\nu}[(2 n+1)(n-\nu) ! /\{4 \pi(n+\nu) !\}]^{1 / 2} \\
& \times P_{\nu}^{n}(\cos \theta) \exp (i \nu \chi), \\
P_{\nu}^{n}(x)= & \left(1-x^{2}\right)^{\nu / 2}\left\{d^{\nu} P_{n}(x) / d x^{\nu}\right\}, \\
P_{-\nu}^{n}(x)= & (-1)^{\nu}\{(n-\nu) ! /(n+\nu) !\} P_{\nu}^{n}(x) .
\end{aligned}
$$

Here, $Y_{n}^{\nu}(\theta, \chi)$ is the spherical harmonics, $P_{\nu}^{n}(x)$ is the Legendre function, and $P_{n}(x)$ is the Legendre polynomial. $R_{0, \nu}^{n}(\theta, \chi)$ has the orthogonality expressed as

$$
\begin{aligned}
& \iint \int R_{0, \mu}^{m}{ }^{*}(\theta, \chi) R_{0, \nu}^{n}(\theta, \chi) \sin \theta d \theta d \phi d \chi \\
& \quad=(-1)^{\mu} \iiint R_{0,-\mu}^{m}{ }^{*}(\theta, \chi) R_{0, \nu}^{n}(\theta, \chi) \sin \theta d \theta d \phi d \chi \\
& \quad=8 \pi^{2} \delta_{m, n} \delta_{\mu, \nu} /(2 n+1) .
\end{aligned}
$$

J. Chem. Phys. 124, 024512 (2006)
The leading projection $g_{00}^{000}(r)$ is the orientationally averaged radial distribution function and represents the solutewater correlation in terms of the molecular centers. The other projections $g_{0 \nu}^{0 n n}(r)=h_{0 \nu}^{0 n n}(r)(n \neq 0)$ represent the solute-water orientational correlation at $r$.

The functions other than the pair-correlation function are also expanded in a similar manner. ${ }^{31-38}$ Substituting Eq. (4) into Eq. (1) and utilizing the orthogonality of $R_{0, \nu}^{n}(\theta, \chi)$ yield

$$
\begin{aligned}
\Delta S^{(2)} / k_{\mathrm{B}}= & \Delta S_{T} / k_{B}+\Delta S_{R} / k_{B}, \\
\Delta S_{T} / k_{\mathrm{B}}= & -4 \pi \rho \int_{0}^{\infty}\left[g_{00}^{000}(r)\left\{-u_{00}^{000}(r) /\left(k_{\mathrm{B}} T\right)\right\}+g_{00}^{000}(r)\right. \\
& \left.\times\left\{h_{00}^{000}(r)-c_{00}^{000}(r)+b_{00}^{000}(r)\right\}\right] r^{2} d r, \\
\Delta S_{R} / k_{\mathrm{B}}= & -4 \pi \rho \sum_{n, \nu}\{1 /(2 n+1)\} \int_{0}^{\infty}\left[g_{0 \nu}^{0 n n}(r)\right. \\
& \times\left\{-u_{0 \nu}^{0 n n}(r) /\left(k_{\mathrm{B}} T\right)\right\}+g_{0 \nu}^{0 n n}(r)\left\{h_{0 \nu}^{0 n n}(r)-c_{0 \nu}^{0 n n}(r)\right. \\
& \left.\left.+b_{0 \nu}^{0 n n}(r)\right\}\right] r^{2} d r, \quad n=1,2, \ldots ; \quad \nu=-n, \ldots, n .
\end{aligned}
$$

Here, $\Delta S_{T}$ and $\Delta S_{R}$ represent the translational and orientational contributions, respectively. We note that since $\exp \left\{u_{00}^{000}(r) /\left(k_{\mathrm{B}} T\right)\right\} g_{00}^{000}(r)$ is finite, $g_{00}^{000}(r)\left\{-u_{00}^{000}(r) /\left(k_{\mathrm{B}} T\right)\right\}$ is zero inside the core. For $n \neq 0, g_{0 v}^{0 n n}(r)=h_{0 v}^{0 n n}(r)$ for all $r$. Applying Eqs. (9) and (10) to the present model system where the solute-water repulsive interaction is the hard-sphere potential yields

$$
\begin{aligned}
\Delta S_{T} / k_{\mathrm{B}}= & -4 \pi \rho \int_{d_{M S}}^{\infty} g_{00}^{000}(r)\left\{h_{00}^{000}(r)-c_{00}^{000}(r)\right. \\
& \left.+b_{00}^{000}(r)\right\} r^{2} d r \\
\Delta S_{R} / k_{\mathrm{B}}= & -4 \pi \rho \sum_{n, \nu}\{1 /(2 n+1)\} \int_{d_{M S}}^{\infty} h_{0 \nu}^{0 n n}(r)\left\{h_{0 \nu}^{0 n n}(r)\right. \\
& \left.-c_{0 \nu}^{0 n n}(r)+b_{0 \nu}^{0 n n}(r)\right\} r^{2} d r, \\
& n=1,2, \ldots ; \quad \nu=-n, \ldots, n .
\end{aligned}
$$

In our model system, $g_{0 \nu}^{0 n n}(r)=0$ for $r<d_{M S}=\left(d_{M}\right.$ $\left.+d_{S}\right) / 2$, and the integration is performed from $d_{M S}$ to $\infty$. The percentage of the orientational contribution $I_{R}$ is given as

$$
I_{R}=100 \Delta S_{R} / \Delta S^{(2)} .
$$

To analyze the orientational order of water molecules near the solute, we calculate the mean value of $P_{2}(\cos \theta)$, $\left\langle P_{2}(\cos \theta)\right\rangle$, which is obtained from

$$
\left\langle P_{2}(\cos \theta)\right\rangle=h_{00}^{022}(r) /\left\{5 g_{00}^{000}(r)\right\} .
$$

Hereafter, $\left\langle P_{2}(\cos \theta)\right\rangle$ is referred to as the order parameter whose absolute value represents the strength of the orientational order per water molecule.

By solving the basic equations derived from the $\mathrm{OZ}$ relation and the closure equation using our efficient algorithm, ${ }^{39-41}$ we can calculate $g_{0 \nu}^{0 n n}(r)$, the translational and orientational contributions, and $I_{R}$. To perform the numerical calculations, the terms for $n \leqslant 4$ are considered in the expan- 
TABLE I. Translational and orientational contributions to the solute-water pair-correlation entropy calculated for the case without the attractive tail $(\varepsilon=0$ and $\lambda=0)$.

\begin{tabular}{cccc}
\hline \hline$d_{M} / d_{S}$ & $-\Delta S_{T}$ (e.u.) & $-\Delta S_{R}$ (e.u.) & $-\Delta S^{(2)}($ e.u. $)$ \\
\hline 0.6 & 1.51 & 1.48 & 2.99 \\
1 & 2.02 & 2.53 & 4.55 \\
1.4 & 2.57 & 3.69 & 6.27 \\
2 & 3.64 & 5.36 & 9.00 \\
3 & 5.91 & 8.13 & 14.0 \\
5 & 13.3 & 14.7 & 28.0 \\
10 & 44.9 & 40.4 & 85.3 \\
15 & 95.6 & 79.4 & 175 \\
20 & 166 & 132 & 297 \\
30 & 363 & 276 & 639 \\
\hline \hline
\end{tabular}

sion based on Wigner's generalized spherical harmonics. ${ }^{31-41}$ The whole calculation can be finished, even for the largest solute $\left(d_{M}=30 d_{S}\right)$, in less than $1 \mathrm{~min}$ on our workstation. It should be noted that our theory is quite general and solutes with significantly large partial charges (i.e., polar solutes) can also be treated. When a reliable bridge function is available, it can be incorporated in the closure equation. In the present study, however, $b_{0 \nu}^{0 n n}(r)$ is set at 0 (the HNC approximation).

\section{RESULTS AND DISCUSSION}

\section{A. Translational and orientational contributions for $d_{M}=d_{S}$}

The translational and orientational contributions to the solute-water pair-correlation entropy calculated for a hardsphere solute $(\varepsilon=0)$ with $d_{M}=d_{S}$ are as follows: $-\Delta S_{T}$ $=2.02$ e.u., $\quad-\Delta S_{R}=2.53$ e.u., $-\Delta S^{(2)}=4.55$ e.u., and $I_{R}$ $=55.6 \%[1$ e.u. $=1 \mathrm{cal} /(\mathrm{mol} \mathrm{K})]$. When the van der Waals attractive tail is incorporated in the solute-water potential, both $-\Delta S_{R}$ and $-\Delta S_{T}$ increase, but $I_{R}$ decreases. In the case of $\varepsilon=0.10 \mathrm{kcal} / \mathrm{mol}$, for example, the quantities calculated are as follows: $-\Delta S_{T}=4.64$ e.u., $-\Delta S_{R}=2.92$ e.u., $-\Delta S^{(2)}$ $=7.56$ e.u., and $I_{R}=38.6 \%$. The values of $I_{R}$ reported by Lazaridis, Ashbaugh, and Paulaitis ${ }^{23,25,30}$ are in the range of $31 \%-42 \%$. Since the van der Waals attractive interaction is included in their work, we can conclude that our result for $I_{R}$ is in good agreement with theirs. The experimental value of the hydration entropy of neon, whose effective diameter is fairly close to $d_{S}$, is -10 e.u. ${ }^{30}$ Our calculated values are fairly close but smaller, which might be attributable to the neglect of the triplet and higher-order solute-water correlations and the water reorganization term.

\section{B. Effects due to solute size and solute-water attractive interaction}

The calculated results are collected in Table I $(\varepsilon=0$ and $\lambda=0$; in the case without the attractive tail) and Table II $[\varepsilon$ $=0.1 \mathrm{kcal} / \mathrm{mol}\left(d_{M} \leqslant 3 d_{S}\right)$ and $\left.\lambda=0.1 \mathrm{kcal} / \mathrm{mol}\left(d_{M} \geqslant 3 d_{S}\right)\right]$. As the solute size increases, both $-\Delta S_{T}$ and $-\Delta S_{R}$ become progressively larger, indicating that the translational and orientational movements of water molecules are more restricted. The two contributions also increase considerably as
TABLE II. Translational and orientational contributions to the solute-water pair-correlation entropy calculated for the case with the attractive tail $\left[\varepsilon=0.1 \mathrm{kcal} / \mathrm{mol}\left(d_{M} \leqslant 3 d_{S}\right)\right.$ and $\left.\lambda=0.1 \mathrm{kcal} / \mathrm{mol}\left(d_{M} \geqslant 3 d_{S}\right)\right]$.

\begin{tabular}{cccc}
\hline \hline$d_{M} / d_{S}$ & $-\Delta S_{T}$ (e.u.) & $-\Delta S_{R}$ (e.u.) & $-\Delta S^{(2)}($ e.u. $)$ \\
\hline 0.6 & 2.92 & 1.61 & 4.53 \\
1 & 4.64 & 2.92 & 7.56 \\
1.4 & 6.45 & 4.40 & 10.9 \\
2 & 10.4 & 6.84 & 17.3 \\
$3^{\text {a }}$ & 19.4 & 10.8 & 30.2 \\
$3^{\mathrm{b}}$ & 17.5 & 10.8 & 28.3 \\
5 & 44.8 & 20.7 & 65.5 \\
10 & 199 & 60.3 & 259 \\
15 & 527 & 121 & 649 \\
20 & 1097 & 203 & 1300 \\
30 & 3219 & 431 & 3649 \\
\hline \hline
\end{tabular}

${ }^{\mathrm{a}} \varepsilon=0.1 \mathrm{kcal} / \mathrm{mol}$ with Eq. (2).

${ }^{\mathrm{b}} \lambda=0.1 \mathrm{kcal} / \mathrm{mol}$ with Eq. (3).

the solute-water attractive interaction becomes stronger. Here, we look at the percentage of the orientational contribution. The solute-size dependence of $I_{R}$ observed in the cases without and with the attractive tail is illustrated in Fig. 1.

First, we discuss the result for $\varepsilon=0$ and $\lambda=0$. As $d_{M}$ becomes larger, the percentage of the orientational contribution $I_{R}$ first increases, takes a maximum value at $d_{M} / d_{S} \sim 2$, and then decreases toward a limiting value $\sim 40 \%$. The percentage reduces considerably as the solute-water attractive interaction becomes stronger. The order parameter, $\left\langle P_{2}(\cos \theta)\right\rangle$, is plotted for five representative values of $d_{M} / d_{S}$ in Fig. 2. It is observed that the orientational order of water molecules near the contact position is the strongest for $d_{M} / d_{S} \sim 2$. Another feature is that the orientational order is short ranged and it becomes only slightly longer ranged as the solute size increases.

Next, the result for $\varepsilon=0.1 \mathrm{kcal} / \mathrm{mol}\left(d_{M} \leqslant 3 d_{S}\right)$ and $\lambda$ $=0.1 \mathrm{kcal} / \mathrm{mol}\left(d_{M} \geqslant 3 d_{S}\right)$ is discussed. We have tested several values of $\varepsilon$ and $\lambda$ which are in the range of $0-0.1 \mathrm{kcal} / \mathrm{mol}$ but present only the result for the largest

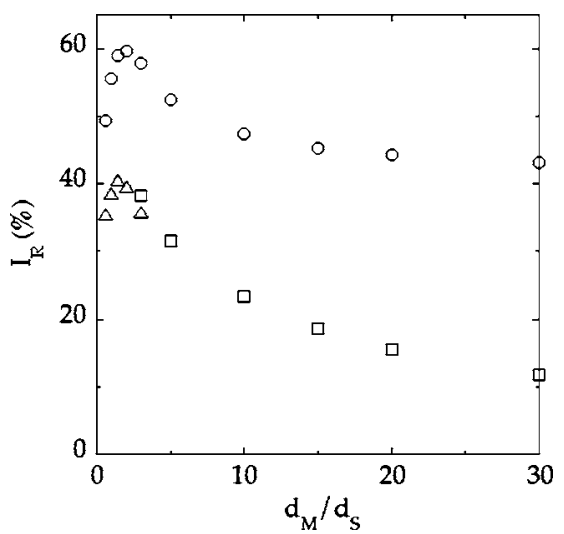

FIG. 1. Percentage of the orientational contribution to the solute-water paircorrelation entropy $\left(I_{R}\right)$ plotted against the solute size $\left(d_{M} / d_{S}\right)$. It is shown for $\varepsilon=0$ and $\lambda=0$ (circles: without the solute-water attractive tail), for $\varepsilon$ $=0.1 \mathrm{kcal} / \mathrm{mol}$ and $d_{M} \leqslant 3 d_{S}$ [triangles: with the solute-water attractive tail given by Eq. (2)], and for $\lambda=0.1 \mathrm{kcal} / \mathrm{mol}$ and $d_{M} \geqslant 3 d_{S}$ [squares: with the solute-water attractive tail given by Eq. (3)]. 


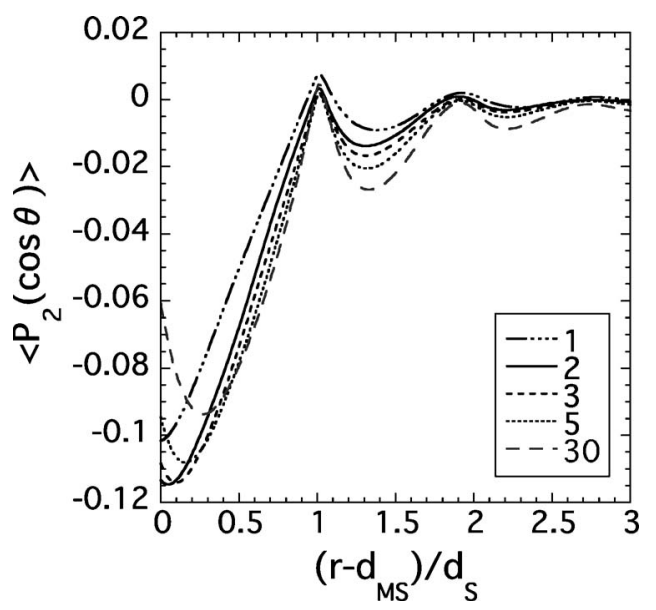

FIG. 2. Order parameter $\left[\left\langle P_{2}(\cos \theta)\right\rangle\right]$ calculated for the five representative solute sizes, $d_{M} / d_{S}=1,2,3,5$, and 30 . The solute-water attractive tail is not incorporated $(\varepsilon=0$ and $\lambda=0)$.

values. Though the solute-size dependence of $I_{R}$ is qualitatively similar to that in the case without the attractive tail, $I_{R}$ is considerably smaller, as shown in Fig. 1. The position of the maximum value of $I_{R}$ shifts to $d_{M} / d_{S} \sim 1.4$. Lazaridis and Paulaitis ${ }^{30}$ reported that the magnitude of the orientational entropy per water molecule in the first hydration shell exhibits a maximum at $d_{M} / d_{S} \sim 1.4$ (they included the van der Waals attractive potential in the solute-water interaction). They performed MC simulations for the TIP4P model of water. Despite the difference in the water models, our result is in complete accord with their observation.

As $\varepsilon$ and $\lambda$ become larger, both $-\Delta S_{T}$ and $-\Delta S_{R}$ increase, but the increase in the former is dominant. When $\kappa$ in Eq. (3) is set at a smaller value and the attractive interaction becomes longer ranged, $-\Delta S_{T}$ increases considerably while $-\Delta S_{R}$ remains almost unchanged. The restriction of the translational movement of water molecules is influenced by the solute-water attractive tail up to fairly large separations, whereas the orientational restriction is sensitive only to the attraction in the close vicinity of the solute. For large solutes, with a sufficiently strong attractive interaction, $-\Delta S_{T}$ is much more important than $-\Delta S_{R}$ (the smallest value of $I_{R}$ in Fig. 1 is $\sim 10 \%$ ).

\section{Restriction of orientational (rotational) freedom of water molecules}

Here, we discuss the physical origin of the maximum orientational restriction observed at $d_{M} / d_{S} \sim 2$. Though the orientational structure of water near a nonpolar solute or an extended hydrophobic surface was discussed in detail by many authors, ${ }^{18-22,30,37,42-45}$ a systematic treatment over a wide range of solute size is made possible only by the integral equation theory. ${ }^{38,46}$ Since the water model employed in the present study has the tetrahedral symmetry, there are no differences between the $\mathrm{OH}$ bond and $\mathrm{O}$ lone pair vectors and they are all referred to as OH-bond vectors. The major quantity containing the information on the solute-water orientational correlation is the probability density $p(\varphi)$ where $\varphi$ is the angle with respect to the surface normal vector (i.e., the vector that is normal to the solute surface) made by either

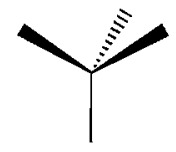

(1)
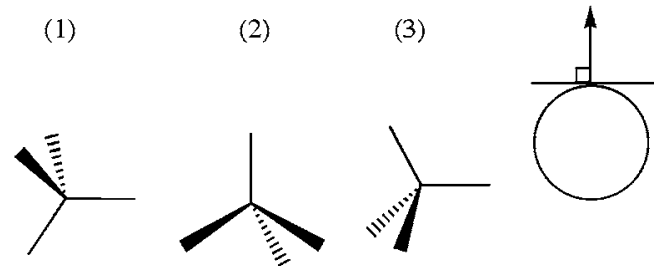

(4)

(5)

(6)

FIG. 3. Six typical orientations of water molecules. The arrow indicates the vector that is normal to the solute surface. The center of the water molecule is at the center of the regular tetrahedron.

the dipole-moment vector $\left(\varphi=\theta_{\mu}\right)$ or the $\mathrm{OH}$ bond $(\varphi$ $\left.=\theta_{\mathrm{OH}}\right)$ for molecules at a given distance from the solute surface. We consider the six typical orientations (1-6) of water molecules illustrated in Fig. 3. Orientations 5 and 6 are, respectively, the inverted counterparts of orientations 1 and 4 (orientations 2 and 3 are symmetric with respect to the inversion). The values of $\theta_{\mathrm{OH}}$ and $\theta_{\mu}$ for the six orientations are given in Table III.

The probability densities $p\left(\theta_{\mathrm{OH}}\right)$ at $\left(r-d_{M S}\right) / d_{S}=0$, $0.33(\sim 1 / 3)$, and $0.67(\sim 2 / 3)$ in the case of no solute-water attractive tail are shown in Figs. 4-6. The densities calculated for five representative values of $d_{M} / d_{S}$ are compared in the figures. First, we review the orientational structure ${ }^{37,38}$ for the two extreme solute sizes, $d_{M} / d_{S}=1$ and 30. At $(r$ $\left.-d_{M S}\right) / d_{S}=0$ (the contact position), for $d_{M} / d_{S}=1$, orientations 5 and 2 are dominant with a very little chance of orientation 1. For $d_{M} / d_{S}=30$, orientations 1,4 , and 3 are dominant while orientations 2,5 , and 6 are not likely. The qualitative aspects of $p\left(\theta_{\mathrm{OH}}\right)$ for $d_{M} / d_{S}=1$ remain unchanged at the three different separations. By contrast, for $d_{M} / d_{S}$ $=30$, a characteristic inversion of $p\left(\theta_{\mathrm{OH}}\right)$ occurs at $(r$ $\left.-d_{M S}\right) / d_{S}=0.33$ beyond which orientations 5,6 , and 3 are dominant and orientations 2, 1 , and 4 are not likely. When a small hydrophobic solute intrudes into water, the water structure neighboring the solute can reorganize by forming the

TABLE III. Angles with respect to surface normal for the typical orientations shown in Fig. 3. Orientations 5 and 6 are, respectively, the inverted counterparts of orientations 1 and 4 . It should be noted that $\theta_{\mu}=55^{\circ}$ is common to orientations 1 and 5 and $\theta_{\mu}=66^{\circ}$ is common to orientations 4 and 6 .

\begin{tabular}{ccccccc}
\hline \hline Orientation & 1 & 2 & 3 & 4 & 5 & 6 \\
\hline \multirow{2}{*}{$\theta_{\mathrm{OH}}$} & $71^{\circ}$ & $55^{\circ}$ & $35^{\circ}$ & $62^{\circ}$ & $0^{\circ}$ & $19^{\circ}$ \\
& $71^{\circ}$ & $55^{\circ}$ & $90^{\circ}$ & $62^{\circ}$ & $109^{\circ}$ & $90^{\circ}$ \\
& $71^{\circ}$ & $125^{\circ}$ & $90^{\circ}$ & $90^{\circ}$ & $109^{\circ}$ & $118^{\circ}$ \\
& $180^{\circ}$ & $125^{\circ}$ & $145^{\circ}$ & $161^{\circ}$ & $109^{\circ}$ & $118^{\circ}$ \\
& & & & & & \\
& $55^{\circ}$ & $0^{\circ}$ & $45^{\circ}$ & $35^{\circ}$ & $55^{\circ}$ & $35^{\circ}$ \\
& $55^{\circ}$ & $90^{\circ}$ & $45^{\circ}$ & $66^{\circ}$ & $55^{\circ}$ & $66^{\circ}$ \\
& $55^{\circ}$ & $90^{\circ}$ & $90^{\circ}$ & $66^{\circ}$ & $55^{\circ}$ & $66^{\circ}$ \\
& $125^{\circ}$ & $90^{\circ}$ & $90^{\circ}$ & $114^{\circ}$ & $125^{\circ}$ & $114^{\circ}$ \\
& $125^{\circ}$ & $90^{\circ}$ & $135^{\circ}$ & $114^{\circ}$ & $125^{\circ}$ & $114^{\circ}$ \\
& $125^{\circ}$ & $180^{\circ}$ & $135^{\circ}$ & $145^{\circ}$ & $125^{\circ}$ & $145^{\circ}$ \\
\hline \hline
\end{tabular}




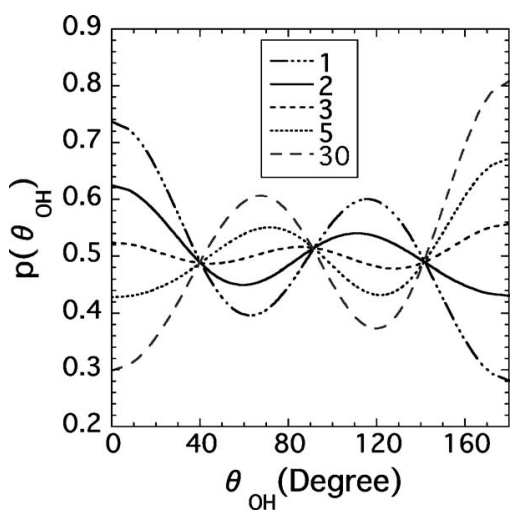

FIG. 4. Probability densities $p\left(\theta_{\mathrm{OH}}\right)$ of water molecules at $\left(r-d_{M S}\right) / d_{S}=0$ calculated for the five representative solute sizes, $d_{M} / d_{S}=1,2,3,5$, and 30 .

clathratelike geometry without sacrificing the intermolecular hydrogen bonds. When a large hydrophobic solute intrudes into water, however, it is impossible to maintain the hydrogen bonds in a similar way and the loss of the bonds is unavoidable. The loss is kept minimal by taking the special orientations.

We now discuss the response of the orientational structure of water molecules near the contact position to the increase in the solute size from $d_{M} / d_{S}=1$. As observed in Fig. 4 , the feature of $p\left(\theta_{\mathrm{OH}}\right)$ persists up to $d_{M} / d_{S} \sim 2$. Beyond $d_{M} / d_{S} \sim 2$, the solute is too large for the water molecules to maintain qualitatively the same orientational structure characterized by orientations 5 and 2 . In orientation 5 , for example, to maximize the number of hydrogen bonds near the contact position, the space surrounded by the four water molecules (i.e., the molecule at the center three molecules at vertices oriented by the $\mathrm{OH}-$ bond vectors with $\theta_{\mathrm{OH}}=109^{\circ}$ ) must accommodate the solute. ${ }^{43}$ This cannot be made possible when the solute is too large. The structure for $d_{M} / d_{S}$ $=5$ is qualitatively similar to that for $d_{M} / d_{S}=30$ and characterized by orientations 1,4 , and 3 . The structural transition occurs at a solute size in the range $2<d_{M} / d_{S}<3$. Thus, $d_{M} / d_{S} \sim 2$ is the largest solute size that allows the water molecules to take orientations 5 and 2. For the small solute of $d_{M} / d_{S}=1$, the restriction of the orientational freedom is not as large as for the solute of $d_{M} / d_{S}=2$. The physical origin of the maximum orientational restriction observed at $d_{M} / d_{S}$

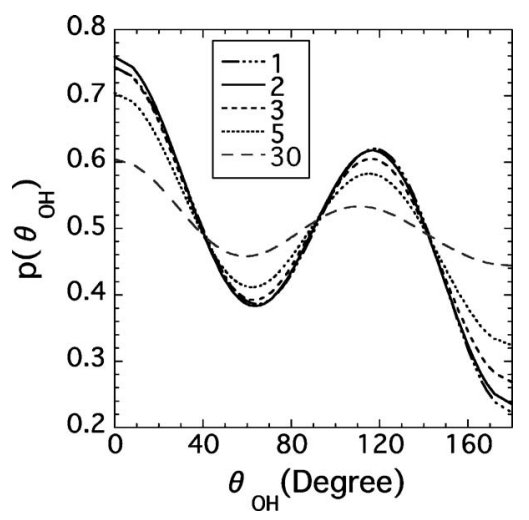

FIG. 5. Probability densities $p\left(\theta_{\mathrm{OH}}\right)$ of water molecules at $\left(r-d_{M S}\right) / d_{S}$ $=0.33$ calculated for the five representative solute sizes, $d_{M} / d_{S}=1,2,3,5$, and 30 .

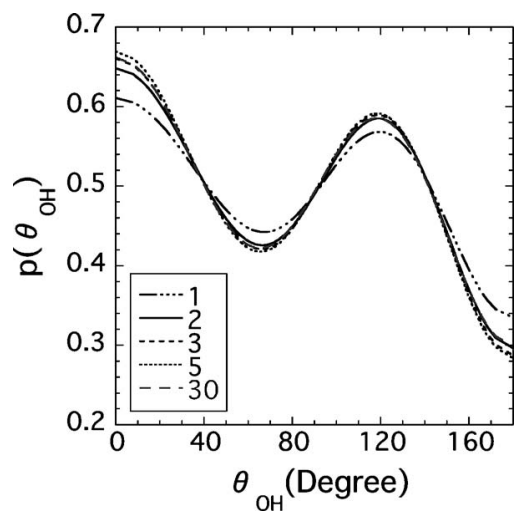

FIG. 6. Probability densities $p\left(\theta_{\mathrm{OH}}\right)$ of water molecules at $\left(r-d_{M S}\right) / d_{S}$ $=0.67$ calculated for the five representative solute sizes, $d_{M} / d_{S}=1,2,3,5$, and 30 .

$\sim 2$ can thus be understood. When the solute-water attractive interaction is present, the four water molecules in orientation 5 or the three water molecules in orientation 2 are attracted to the solute and in contact with it in much higher probability. As a result, the size of the solute that the space can accommodate becomes slightly smaller.

\section{CONCLUDING REMARKS}

The hydration properties of a nonpolar solute are characterized by the restrictions of the translational and orientational movements of water molecules upon solute insertion, causing a large entropic loss. We have developed an efficient method which enables us to evaluate the relative magnitudes of the contributions from the translational and orientational restrictions to the solute-water pair-correlation entropy, a major component of the hydration entropy. The angle-dependent Ornstein-Zernike integral equation theory ${ }^{31-38}$ is applied to the statistical-mechanical formulation previously derived by Lazaridis, Ashbaugh, and Paulaitis, ${ }^{23,25,30}$ and by Matubayasi et $a .^{26}$ All the correlation functions are expanded using Wigner's generalized spherical harmonics. By utilizing their orthogonality, the pair-correlation entropy is decomposed into the two contributions in an analytical manner without using the factorization assumption. The basic equations are solved using our rapidly convergent algorithm. ${ }^{39-41}$

The translational and orientational contributions have been calculated for solutes with a variety of sizes (0.6 $\left.\leqslant d_{M} / d_{S} \leqslant 30\right)$. The effects of the solute-water attractive interaction are also studied. The orientational restriction is always much shorter ranged than the translational one. As $d_{M}$ becomes larger, the percentage of the orientational contribution first increases, takes a maximum value at $d_{M}=D_{M}$, and then decreases toward a limiting value. $D_{M} / d_{S}$ is $\sim 2$ in the case of no solute-water attractive interaction. $D_{M} / d_{S}$ becomes slightly smaller when the attractive interaction is incorporated, and $D_{M} / d_{S} \sim 1.4$ for the strongest attraction tested. By analyzing the orientational structure of water near the solute, we have clarified the physical origin of the largest restriction of orientational freedom of water molecules. The percentage of the orientational contribution reduces progressively as the solute-water attractive interaction becomes stronger. Though the translational and orientational contribu- 
tions are equally important for small solutes, the former is dominant for large solutes with a sufficiently strong solutewater attraction.

Since the translational and orientational contributions depend on the solute-water interaction potential and the temperature differently, the hydration entropy of a large solute and that of a small solute should behave differently against the changes in these parameters. The effects of the temperature for nonpolar solutes with a variety of sizes are to be investigated. Further, this kind of analysis is to be extended to a polar solute. Works in these directions are in progress.

\section{ACKNOWLEDGMENTS}

This work was supported by Grants-in-Aid for Scientific Research on Priority Areas (Grant Nos. 15076203 and 15076205) from the Ministry of Education, Culture, Sports, Science and Technology of Japan and by NAREGI Nanoscience Project.

${ }^{1}$ R. Levy and E. Gallicchio, Annu. Rev. Phys. Chem. 49, 531 (1998).

${ }^{2}$ L. R. Pratt, Annu. Rev. Phys. Chem. 53, 409 (2002).

${ }^{3}$ R. A. Pierotti, Chem. Rev. (Washington, D.C.) 76, 717 (1976).

${ }^{4}$ B. Lee, Biopolymers 24, 813 (1985).

${ }^{5}$ R. L. Baldwin, Proc. Natl. Acad. Sci. U.S.A. 83, 8069 (1986)

${ }^{6}$ D. E. Smith and A. D. J. Haymet, J. Chem. Phys. 98, 6445 (1993).

${ }^{7}$ B. Madan and B. Lee, Biophys. Chem. 51, 279 (1994).

${ }^{8}$ A. Wallqvist and B. J. Berne, J. Phys. Chem. 99, 2885 (1995).

${ }^{9}$ G. Hummer, S. Garde, A. E. García, A. Pohorille, and L. R. Pratt, Proc. Natl. Acad. Sci. U.S.A. 93, 8951 (1996).

${ }^{10}$ M. Ikeguchi, S. Shimizu, S. Nakamura, and K. Shimizu, J. Phys. Chem. B 102, 5891 (1998).

${ }^{11}$ R. D. Mountain and D. Thirumalai, Proc. Natl. Acad. Sci. U.S.A. 95, 8436 (1998)

${ }^{12}$ K. A. T. Silverstein, A. D. J. Haymet, and K. A. Dill, J. Chem. Phys. 111, 8000 (1999)

${ }^{13}$ P. L. Chan and R. L. Mancera, Mol. Phys. 96, 109 (1999).
${ }^{14}$ K. Lum, D. Chandler, and J. D. Weeks, J. Phys. Chem. B 103, 4570 (1999).

${ }^{15}$ V. A. Parsegian, R. P. Rand, and D. C. Rau, Proc. Natl. Acad. Sci. U.S.A. 97, 3987 (2000).

${ }^{16}$ H. S. Frank and M. W. Evans, J. Chem. Phys. 13, 507 (1945).

${ }^{17}$ J. L. Finney and A. K. Soper, Chem. Soc. Rev. 23, 1 (1994).

${ }^{18}$ G. Alagona and A. Tani, J. Chem. Phys. 72, 580 (1980).

${ }^{19}$ A. Geiger, A. Rahman, and F. H. Stillinger, J. Chem. Phys. 70, 263 (1979).

${ }^{20}$ C. Pangali, M. Rao, and B. J. Berne, J. Chem. Phys. 71, 2982 (1979).

${ }^{21}$ P. J. Rossky and M. Karplus, J. Am. Chem. Soc. 101, 1913 (1979).

${ }^{22}$ P. J. Rossky and D. A. Zichi, Faraday Symp. Chem. Soc. 17, 69 (1982).

${ }^{23}$ T. Lazaridis and M. E. Paulaitis, J. Phys. Chem. 96, 3847 (1992).

${ }^{24}$ H. Yu and M. Karplus, J. Chem. Phys. 89, 2366 (1988).

${ }^{25}$ H. S. Ashbaugh and M. E. Paulaitis, J. Phys. Chem. 100, 1900 (1996).

${ }^{26}$ N. Matubayasi, L. H. Reed, and R. M. Levy, J. Phys. Chem. 98, 10640 (1994).

${ }^{27}$ N. Matubayasi and R. M. Levy, J. Phys. Chem. 100, 2681 (1996).

${ }^{28}$ T. Lazaridis, J. Phys. Chem. B 102, 3531 (1998).

${ }^{29}$ T. Lazaridis, J. Phys. Chem. B 102, 3542 (1998).

${ }^{30}$ T. Lazaridis and M. E. Paulaitis, J. Phys. Chem. 98, 635 (1994).

${ }^{31}$ P. H. Fries and G. N. Patey, J. Chem. Phys. 82, 429 (1985).

${ }^{32}$ P. G. Kusalik and G. N. Patey, J. Chem. Phys. 88, 7715 (1988).

${ }^{33}$ P. G. Kusalik and G. N. Patey, Mol. Phys. 65, 1105 (1988).

${ }^{34}$ G. M. Torrie, P. G. Kusalik, and G. N. Patey, J. Chem. Phys. 88, 7826 (1988).

${ }^{35}$ D. R. Bérard and G. N. Patey, J. Chem. Phys. 95, 5281 (1991).

${ }^{36}$ M. Kinoshita, S. Iba, and M. Harada, J. Chem. Phys. 105, 2487 (1996).

${ }^{37}$ M. Kinoshita, J. Solution Chem. 33, 661 (2004).

${ }^{38}$ M. Kinoshita, J. Mol. Liq. 119, 47 (2005).

${ }^{39}$ M. Kinoshita and M. Harada, Mol. Phys. 79, 145 (1993).

${ }^{40}$ M. Kinoshita and M. Harada, Mol. Phys. 81, 1473 (1994).

${ }^{41}$ M. Kinoshita and D. R. Bérard, J. Comput. Phys. 124, 230 (1996).

${ }^{42}$ C. Y. Lee, J. A. McCammon, and P. J. Rossky, J. Chem. Phys. 80, 4448 (1984).

${ }^{43}$ N. Matubayasi, J. Am. Chem. Soc. 116, 1450 (1994).

${ }^{44}$ M. Kinoshita and F. Hirata, J. Chem. Phys. 104, 8807 (1996).

${ }^{45}$ D. M. Huang, P. L. Geissler, and D. Chandler, J. Phys. Chem. B 105, 6704 (2001).

${ }^{46}$ N. M. Cann and G. N. Patey, J. Chem. Phys. 106, 8165 (1997). 